\title{
Latencies in fMRI Time-Series: Effect of Slice Acquisition Order and Perception
}

\author{
Pierre-François Van de Moortele ${ }^{1}$, Barbara Cerf $^{2}$, Elie Lobel ${ }^{1,3}$ Anne-Lise Paradis ${ }^{1,3}$, Annick \\ Faurion $^{2}$ and Denis Le Bihan ${ }^{1}$ \\ ${ }^{1}$ Service Hospitalier Frédéric Joliot, Département de Recherche Médicale, CEA, 4, place du Général Leclerc, 91406 Orsay, France \\ ${ }^{2}$ Labatoire de Neurobiologie Sensorielle de l'Ecole Pratique des Hautes Etudes, 1 Avenue des Olympiades, 91305 Massy, France \\ ${ }^{3}$ Laboratoire de Physiologie et de Perception de l'Action, CNRS at Collège de France, 11 place Marcelin Berthelot, 75231 Paris \\ Cedex, 05, France
}

\begin{abstract}
In BOLD fMRI a detailed analysis of the MRI signal time course sometimes shows time differences between different activated regions. Some researchers have suggested that these latencies could be used to infer the temporal order of activation of these cortical regions. Several effects must be considered, however, before interpreting these latencies. The effect of a slice-dependent time shift (SDTS) with multi-slice acquisitions, for instance, may be important for regions located on different slices. After correction for this SDTS effect the time dispersion between activated regions is significantly decreased and the correlation between the MRI signal time course and the stimulation paradigm is improved. Another effect to consider is the latency which may exist between perception and stimulus presentation. It is shown that the control of perception can be achieved using a finger-spanning technique during the fMRI acquisition. The use of this perception profile rather than an arbitrary waveform derived from the paradigm proves to be a powerful alternative to fMRI data processing, especially with chemical senses studies, when return to baseline is not always correlated to stimulus suppression. This approach should also be relevant to other kinds of stimulation tasks, as a realistic way of monitoring the actual task performance, which may depend on attention, adaptation, fatigue or even variability of stimulus presentation. () 1997 John Wiley \& Sons, Ltd.
\end{abstract}

NMR in Biomed. 10, 230-236 (1997) No. of Figures: 7 No. of Tables: 1 No. of References: 32

Keywords: functional MRI; echo planar imaging; multi-slice; perception; taste

Received 12 March 1997; revised 2 May 1997; accepted 2 May 1997

\section{INTRODUCTION}

An important issue in cognitive neuroscience is to map out the cortical networks which produce brain responses during various conditions, from simple motor tasks to elaborate language or mental imagery activities. It is clear that many cortical regions are involved in these processes and that these regions are highly connected. As a single region, such as the cingular gyrus, may participate to many different kinds of processes, connections between different neuronal systems should be established on a time basis. In this context, neuroimaging has appeared as a method of choice, as it may address both the issues of spatial localization and temporal orchestration of cortical activation. So far, MEG/ EEG or ERP based studies provide the best temporal resolution (down to $1 \mathrm{~ms}$ ), but suffer from poor localization, while PET is associated with good spatial resolution. Functional MRI potentially provides an even better spatial resolution, and an interesting and challenging prospect for this technique is to find out whether it may also give access to a good temporal resolution.

${ }^{\star}$ Correspondence to: Denis Le Bihan; e-mail: lebihan@uriens.shfj.cea.fr. Contract grant sponsor: CNRS ('GIS Cognition')

Abbreviations used: BOLD, blood oxygen level dependent; EEG, electroencephalography; EPI, echo-planar imaging; ERP, event-related potential; fMRI, functional MRI; MC, motor cortex; MEG, magnetoencephalography; PET, positron emission tomography; PP, perception profile SDTS, slice-dependent time shift; SMA, supplementary motor area; SPW, stimulation paradigm waveforms.
Today, most fMRI experimental designs rely on two alternating conditions, usually an 'activated' and a 'control' (sometimes called 'rest') condition, taking advantage of the ability of fast imaging acquisition schemes to follow the relaxation changes which result from the BOLD effect in real time. ${ }^{1,2}$ A detailed analysis of the MRI signal time courses obtained from such studies has sometimes shown time shifts between the BOLD MRI signals observed in different cortical regions. Some researchers have suggested that these time shifts could be used to infer the temporal order of activation of these cortical regions. ${ }^{3-7}$ We have, however, to be extremely careful in assessing these time shifts, as several pitfalls must be avoided. For instance, differences in the structure of the vascular networks which support the activated regions or in their metabolism may result in slightly different signal responses. In this paper, we have looked more specifically to two potential pitfalls, one related to the way the image data is collected, the other, more subtle, to the biological nature of the response.

\section{BACKGROUND}

\section{Effect of the acquisition scheme}

With single slice acquisition schemes ${ }^{4,8,9}$ all pixel signals are stimultaneously collected for each time point. Thus, in these studies it is possible to directly compare the time 
course of the MRI signal responses of the different pixels. This possibility would also hold with true 3D echo planar. ${ }^{6}$ However, multislice EPI has become the most commonly used mode of acquisition in fMRI, as it provides an efficient way to scan the whole brain in a few seconds with limited sensitivity to motion artifacts. With this approach, though, the brain response is sampled slice by slice, with a slicedependent time shift (STDS) with respect to the paradigm directly related to the temporal order of acquisition of the slices. With typical repetition times from 3 to $6 \mathrm{~s}$, one could expect that two activated pixels responding simultaneously, but located in different slices, could show a time difference up to 3 or $6 \mathrm{~s}$ (Fig. 1). If not corrected this effect would hamper the detection of any physiological delays in the range of a few hundred milliseconds.

\section{Perception effect}

A common assumption with BOLD fMRI is that the brain response directly follows the time course of the stimulation paradigm. With this approach, activation foci are most often detected by comparing the mean signal levels between the stimulation and control periods, for instance by correlating pixel time-course series to a waveform derived from stimulation taking into account the hemodynamic response of the fMRI signal. ${ }^{10}$ However, perception, may not always closely follow the stimulation time-course.

With chemical senses, for instance, the perception timecourse might be rather unpredictable from the stimulation paradigm. Hence, data processing algorithms which are satisfactory with other sensory studies such as vision or audition, may drastically fail to detect activation with chemical senses. Taste studies, for instance, have shown that there might be a latency of several seconds between the arrival of a chemical stimulus on the tongue and the beginning of a taste response (Fig. 2). Then, the response may build up very slowly, taking several seconds to reach its maximum. ${ }^{11}$ The latency and rise-time of the response mostly depend on the stimulus nature, but also on individual subjects. The equilibrium between adsorption and desorption is a feature of the relationship between a given tastant

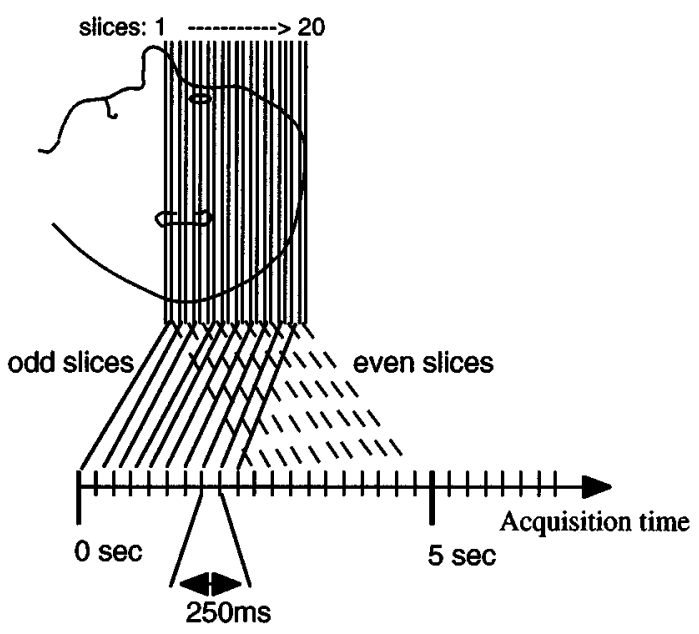

Figure 1. Slice-dependent time shift (SDTS) effect. Example of SDTS effect that could be observed with a scheme of 20 interleaved slices acquired using a $5 \mathrm{~s}$ TR. During each TR interval the delay between the acquisition time of two different slices depends on its rank order in the sequence and may reach up to $4750 \mathrm{~ms}$. With the interleaved scheme lodd then even slices) two adjacent slices are separated by about 2500 ms.

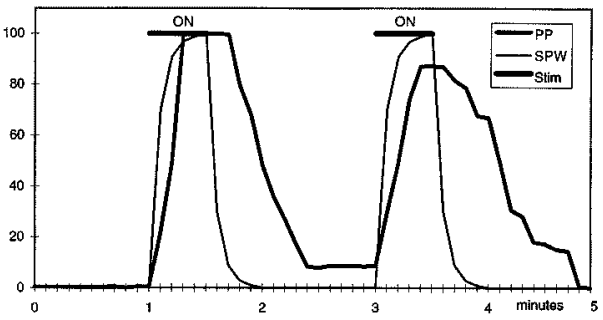

Figure 2. Perception profile and stimulation paradigm waveform in a taste experiment (Aspartame, $0.2 \mathrm{~mm}$ ). The Stimulation Paradigm Waveform (SPW) is derived from the convolution of the stimulation time course (here two ON and three OFF epochs) with a $5 \mathrm{~s}$ time-constant exponential. The perception profile (PP) is recorded for each subject and for each stimulation using a finger-span method. PP represents the temporal evolution of the perceived taste intensity. For this subject there is a clear time lag of the PP with respect to the SPW.

and taste receptor proteins. Due to their hydrophobic nature, some tastants are difficult to rinse with water, a phenomenon which may result in a long-lasting perception lingering up to one minute or more. ${ }^{12}$ Finally, adaptation (both peripheral and central) may result in a fading perception for a constant level of stimulation. ${ }^{13,14}$ Therefore, it is quite impossible to predict the sensation elicited by a particular stimulus for a given subject, because of the great interindividual and inter-molecular variability characterizing the sense of taste. ${ }^{15-17}$ More generally, cognitive factors may also interfere with perception, even at the level of the primary cortices, as these cortices have been shown to be activated by mental imagery. ${ }^{18,19}$

\section{METHODS}

\section{Data acquisition}

The study was approved by a National Ethics Committee for Biomedical Research Experiments and performed using a 3 Tesla whole body MR scanner (Bruker, Germany) equipped with an insert head gradient coil allowing echo-planar imaging. To minimize movement artifacts, the subject head was maintained with foam pads within a bird-cage quadrature rf coil. At the beginning of each session, a scout $T_{1}$-weighted sagittal image was acquired to position slices of interest. High resolution, high contrast inversion-recovery images $(256 \times 256$ pixels, $T R=3 \mathrm{~s}, T E=8 \mathrm{~ms}$, $T I=800 \mathrm{~ms}$ ) were acquired for anatomical identification of the activated foci.

For the SDTS effect the paradigm consisted in a finger tapping task with eight ON-OFF cycles of $30 \mathrm{~s}$. This task was chosen for its robustness and its resulting activation of multiple, widely separated brain regions. A total of 51 blocks of NS $=20$ contiguous axial gradient-echo EPI slices were acquired $(64 \times 64$ pixels, $5 \mathrm{~mm}$ thickness, $22 \mathrm{~cm}$ field of view, $T R=5 \mathrm{~s}, T E=40 \mathrm{~ms}$, interleaved slices).

The feasibility of monitoring perception and using the temporal evolution of the effect perceived by subjects during a taste fMRI study was evaluated using a finger-span approach which is considered as a valid quantitative counterpart of the physiological response. ${ }^{20-22}$ Eight subjects were trained to match the intensity of their perception of a stimulus with a distance between their thumb and first finger, both attached to a linear potentiometer connected 
through a low-pass filter to an analog-digital converter located outside the shielded room. Subjects were asked to continuously match their finger-span to the intensity of their perception. This intensity was thus translated into a variable voltage which was digitized and stored. ${ }^{22}$ The stimulation paradigm consisted in two ON and three OFF periods: a first $60 \mathrm{~s}$ OFF period of reference with water, a $30 \mathrm{~s}$ ON stimulation, a $90 \mathrm{~s}$ OFF water rinse, a $30 \mathrm{~s} \mathrm{ON}$, a $90 \mathrm{~s}$ OFF water rinse. During the $\mathrm{ON}$ period a low concentration tastant solution was delivered directly to the subject's mouth through microsyringes and polyethylene tubing (i.d.: $1.19 \mathrm{~mm}$ ) as bolus of $50 \mu \mathrm{L}$ manually pushed every $3 \mathrm{~s}$. Subjects could swallow freely. Fifty-one blocks of 12 EPI slices located around the Sylvian fissure images $(64 \times 64$ pixels, $6 \mathrm{~mm}$ thickness, $20 \mathrm{~cm}$ field of view, $T R=6 \mathrm{~s}$, $T E=40 \mathrm{~ms}$ ) were continuously recorded for $5 \mathrm{~min}$. Each subject underwent three experiments with various tastants and a control with water.

\section{Data analysis}

Images were processed using a custom software written under IDL (Interactive Data Language, Research System Inc., Boulder, CO). Images were first checked for evidence of head motion artifacts. Evidence of substantial movement implied no further analysis. The first three blocks of images of each series were discarded from analysis, because the magnetization was not steady at the beginning of the experiment. Each pixel signal time course (48 time points) was then low-pass filtered in the time domain with a gaussian kernel of 5 and $8 \mathrm{~s}$ full width at half maximum height for the SDTS and the perception effect, respectively.

For the SDTS effect an auto correlation analysis was first performed to detect activated clusters without any a priori knowledge of any time shift. It was possible to use autocorrelation through the strictly periodic nature of paradigm. An index of activation was defined as $(A C(T)$ $A C(T / 2)) / 2$, where $A C(T)$ and $A C(T / 2)$ are the autocorrelation coefficients obtained by shifting the pixel response curve by one period (here $30 \mathrm{~s}$ ) and half a period, respectively. ${ }^{23}$ Only $3 \mathrm{D}$ clusters larger than three contiguous pixels were considered as activated. These 3D activated clusters, overlapping several contiguous slices, were split into slice subsets (2D activated clusters). The phase, $\mathrm{PhR}$, of the MRI signal response time course with respect to the paradigm waveform was then calculated for each slice subset. PhR was determined from the peak observed at the paradigm frequency $(1 / 30 \mathrm{~Hz})$ after Fourier transform of the average MRI signal time curve of each slice subset. Finally, two cross-correlation coeficients ${ }^{10}$ were calculated for each slide subset: first by correlation with a native paradigm waveform (box car convoluted with a $5 \mathrm{~s}$ time constant exponential, ${ }^{24}$ second after shifting this waveform to correct for the SDTS effect. For this correction, the time shift was calculated as $\Delta t=(T R / N S) *\left(N_{\text {acq }}-1\right)$, where $N S$ is the number of slices and $N_{\text {acq }}$ the temporal order of the slice in the $T R$ interval. Thus, $\Delta t$ ranges from 0 to $4750 \mathrm{~ms}$.

For the perception study activation maps were calculated on a pixel-by-pixel basis for each slice based on the correlation coefficient between the MR signal time course for each pixel, and both the Perception Profile (PP) and a Stimulation Paradigm Waveform (SPW) taking into account the delay in the MRI signal resulting from its hemodynamic nature $^{10}$ (the square paradigm waveform was also convoluted with a gaussian kernel using an $8 \mathrm{~s}$ time constant).
Only the pixels with a correlation coefficient above 0.4 and belonging to clusters of at least 2 contiguous activated pixels were considered for statistical analysis. Then, a Student $t$-test was performed on each individual cluster. The average MRI signal of the images acquired during the control and activated conditions once in the steady-state were compared. Only clusters with a signifcance threshold of $p<0.001$ were finally considered as activated.

\section{RESULTS}

\section{Slice Dependence Time Shift}

During finger tapping activation was clearly visible in areas which have been previously reported, ${ }^{25}$ especially in the primary motor cortex (MC) and the supplementary motor cortex area (SMA). Activation was also seen in the cerebellum and in the parieto-temporal region. In order to analyze the SDTS effect, the three-dimensional clusters corresponding to those regions were split into subsets when they encompassed several slices. Table 1 shows a typical distribution of these slice subsets for one of our subjects. In the plot shown in Fig. 3 the phase of the slice subset response time courses is highly correlated $\left(r^{2}=0.61\right)$ with the chronological order of acquisition of the slice within the $T R$ interval. The slope derived from the linear regression analysis of this plot is $0.25 \mathrm{~s} / \mathrm{image}$, which matches exactly the value that would be expected from the acquisition scheme $(T R / N S=5 / 20 \mathrm{~s} /$ image $)$. After correction for this time shift a significant improvement in the correlation

\begin{tabular}{lccc}
\hline Table 1. Distribution of 23 slice subsets from four 3D clusters \\
of activated pixels. Regions of interest are from \\
cerebellum (CB), temporo-parietal region (TP), sup- \\
plementary motor area (SMA) and motor cortex \\
(MC). Slice position is from bottom to top of brain \\
Size & $\begin{array}{l}\text { Number of } \\
\text { slice position } \\
\text { (pixels) }\end{array}$ & slice subsets & of subsets \\
Cluster & 47 & 5 & $1-5$ \\
location & 24 & 5 & $11-15$ \\
CB & 49 & 7 & $14-20$ \\
TP & 72 & 6 & $15-20$ \\
MC & 192 & 23 & \\
SMA & \multicolumn{4}{l}{} \\
Total & & & \\
\hline
\end{tabular}

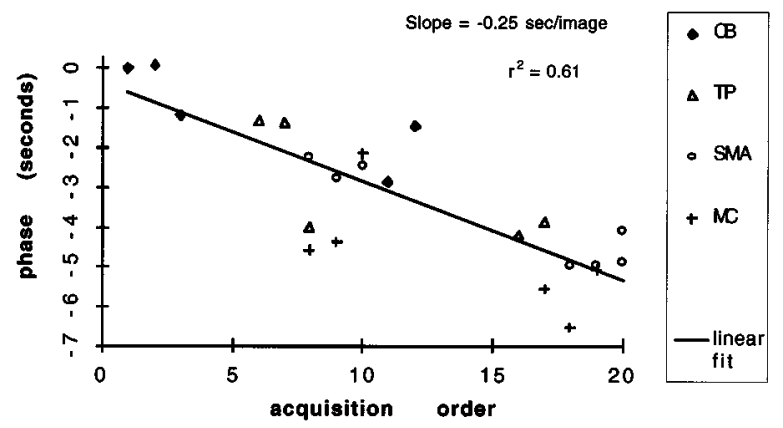

Figure 3. Time shift vs slice acquisition order. Observed response time shifts (in seconds) for the 23 analyzed 2D slice subsets plotted against the chronological order of acquisition of the slice they belong to during TR. The slope derived from a linear regression is $0.250 \mathrm{~s} / \mathrm{image}\left(r^{2}=0.61\right)$. The phase of the first acquired slice has been used as the phase reference. $(\mathrm{CB}=$ cerebellum, $\mathrm{TP}=$ temporo-parietal region, $\mathrm{SMA}=$ supplementary motor area, $\mathrm{MC}=$ motor cortex). 


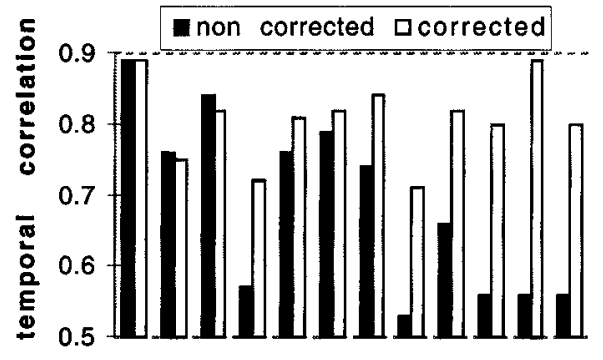

Figure 4. Effect of the SDTS correction on the correlation coefficient. Cross-correlation coefficients calculated for 12 of the 23 slice subsets, before (black) and after (white) SDTS correction, ordered by sequential order of acquisition. The improvement in the correlation coefficients appears clearly for the slices which are acquired late in the TR interval.

coefficient is clearly visible, especially for the slices acquired late in the $T R$ interval (Fig. 4). In the latest slices some subsets present a non-corrected correlation coefficient of about 0.5 rising up to about 0.9 after correction. The correction for the SDTS effect also leads to an overall significant decrease of the phase dispersion of the signal time courses observed between slice subsets (Fig. 5).

\section{Perception effect}

The perception profiles (PP) clearly differ from the usual waveforms derived from a square-wave stimulation paradigm (SPW) (Fig. 2). The PP time-courses obtained with Aspartame are slower than the expected hemodynamic response with a longer rising period (up to $25 \mathrm{~s}$ ), a longer decay and sometimes no return to baseline after each ON period (up to $45 \mathrm{~s}$ ), and vary largely between subjects and experiments (e.g. the second $\mathrm{ON}$ period resulting in a smaller, slower and longer lasting perception response than the first one). The same temporal features appear as well in the MRI signal responses (Fig. 6). In the activation maps significantly more activation foci are found with the PP than the SPW reference waveforms (Fig. 7). Overall, 20 out of 24 experiments showed activation with the PP. The mode of the distribution of the correlation coefficient is higher with the PP than with the SPW. About $30 \%$ of the activated clusters have a correlation coefficient above 0.65 with the PP, while no cluster above 0.65 could be detected with the SPW. Furthermore, the locations of the clusters detected with the PP closely match regions found activated by taste stimulation in electrophysiological studies performed in monkeys. Cortical areas near the Sylvian fissure have been

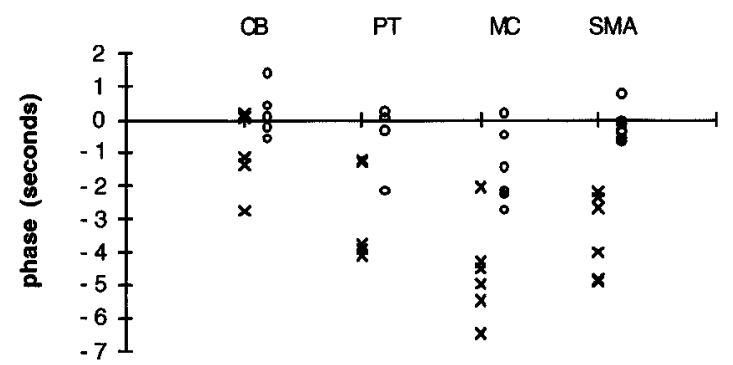

Figure 5. Phase dispersion within clusters before and after SDTS correction. Calculated time shift for each slice subset before (cross) and after (circle) correction for the STDS effect. Slice subsets belonging to the same cluster have been put together. $(C B=$ cerebellum, $\mathrm{TP}=$ temporo-parietal region, SMA= supplementary motor area, $\mathrm{MC}=$ motor cortex). The phase dispersion is reduced within each cluster after correction, also a residual time shift can still be seen.

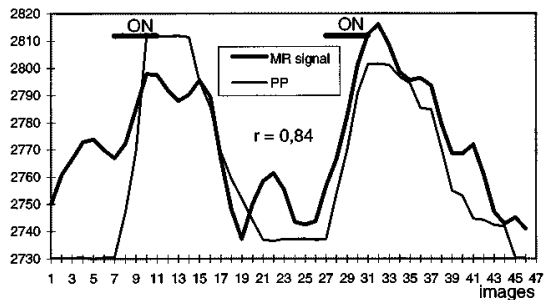

Figure 6. Perception profile and $\mathrm{fMRI}$ response (taste stimulation with Aspartame). The fMRI signal time course of 4 activated voxels in the left insula was temporally smoothed using a $8 \mathrm{~s}$ Gaussian kernel. The fMRI response is clearly correlated with the PP ( $r=0.84$ ) (same subject as in Fig. 2).

proposed as being involved in taste perception. ${ }^{26,27}$ In many of our measurements (15 out of 24 ), clusters of pixels extracted using the taste perception paradigm were found in this region. In the example shown in Fig. 7 an increased activity was observed in the left and right insula, the left and right inferior parts of the pre and post central gyri, and the right operculum. When the SPW was used to process the same data set, the number of activated clusters of pixels significantly dropped and activity could be located only in right insula.

\section{DISCUSSION}

The issue of the phase of the MRI signal time course with respect to the paradigm has been brought up in several important studies. For instance, it has been suggested that within an activated region of interest, additional delays could be observed in the vicinity of larger vessels. ${ }^{8,9}$ Lee $e t$ al. have shown that the time lag of the pixel response could be used to discriminate between 'true' cortical activation and vascular signal variations. ${ }^{9}$ Another interesting idea is to purposely include a time shift between different components present in a single paradigm to detect different activated subregions by means of a phase segregation between the corresponding elicited responses. In this way Sereno et al. have demonstrated the anatomical topography of visual areas in humans. ${ }^{28}$ In this work the authors appropriately removed the effect of the slice acquisition order before analyzing their data. Savoy et al. have shown that by delaying visual presentations towards right and left visual hemifields by $500 \mathrm{~ms}$, the corresponding responses presented similar time shifts. ${ }^{29}$ Hykin et al. have shown similar results between visual stimuli and motor tasks. ${ }^{5}$ Bandettini et al. had previously shown that when cueing different task frequencies for right and left hand in a finger tapping experiment, the corresponding cerebral motor areas exhibited segregated responses in terms of frequencies. ${ }^{7}$

Other exciting physiological results have also been obtained by detecting time shifts between different activated cerebral regions elicited by a single stimulus. Binder et al. showed delays of about one second between elicited temporal activated subregions following auditory stimulation. ${ }^{3}$ In finger tapping experiments Erb et al. have found a time shift of $1.3 \mathrm{~s}$ between SMA and premotor cortex BOLD responses,${ }^{4}$ in good agreement with electrophysiological data. ${ }^{30}$ Hykin et al. found about $2500 \mathrm{~ms}$ between SMA and MC response in finger tapping experiments, ${ }^{5}$ and Mansfield et al. have measured delays up to $660 \mathrm{~ms}$ within visual cortex after visual stimulus presentation. ${ }^{6}$ It should be noted, however, that neither of these studies were performed 
using multi-slice acquisitions.

These studies clearly underline the importance of the analyzes of the time difference that may exist between the MRI response and the paradigm time course. Our results indicate that it is very important to remove the SDTS effect in multi-slice fMRI before assessing any phase variations or temporal delays across activated regions. The correction for the SDTS effect is very straightforward and should always be used to eliminate one source of systematic error. The SDTS effect would be especially significant for clusters which are widely distant in the acquisition. It should be pointed out that this 'time' distance may not coincide with an anatomical distance, as regions located on contiguous slices may not be sequentially acquired in an interleaved acquisition mode. The effect would be more difficult to take into account with more conventional (non EPI) multislice, multishot gradient echo sequences as $k$-space is spanned over time intervals comparable to the time constant of the BOLD response. The time shift to consider would then be that present at the $k$-space center.

Another interest of the correction of the SDTS effect is that it significantly improves the cross-correlation coefficients, especially for slices acquired lastly in $T R$, potentially leading to the detection of more activated regions. This result may also be useful with strategies which determine the maximum value of the correlation coefficient for each pixel by shifting in time the reference waveform. ${ }^{3,7}$
If this strategy is used in multi-slice studies, the SDTS correction will reduce the computing time, especially for long $T R$, providing a smaller range of time shifts to be tested. The improvement in the correlation coefficent would be larger for paradigms with a period comparable to $T R$.

In our study residual phase variations are still visible between some activated regions after SDTS correction (Fig. 5 ). The origin of these differences is still not clear, but could potentially lead to some interesting findings about the chronology of the activation. We calculated the phase of the response time courses using the Fourier transform of the paradigm time course. ${ }^{7}$ Unfortunately, the phase reference could be difficult to determine, as paradigm time courses usualy differ from sinusoid curves and lead to complex shapes after Fourier transform. Similarly, any asymmetry, as usually observed between response rises and decays, would lead to a harmonic enrichment and to some phase alteration of the paradigm frequency. ${ }^{10}$ So, if one wishes to examine very small phase differences, response shapes should be taken into account. In some cases, the sampling rate $(1 / T R)$ should also have to be considered, as it may lead to aliasing for frequencies higher than $1 / 2 T R$. These high frequencies can arise from noise, cardiac cycle, respiratory cycle, movements, other physiologic fluctuations, etc. ${ }^{8}$

Another difficulty in analyzing fMRI time series is that the actual time course of each activated region may differ from the theoretical paradigm time course for physiological

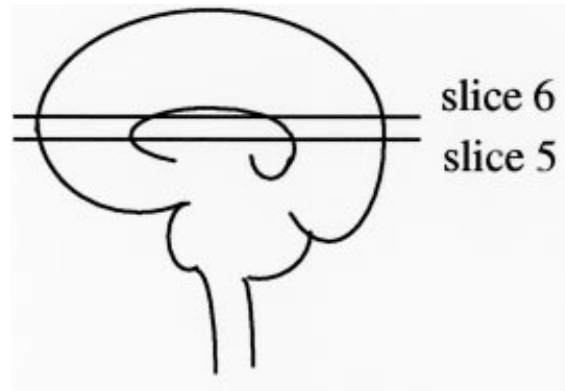

\section{A. SPW processing}

\section{B. PP processing}

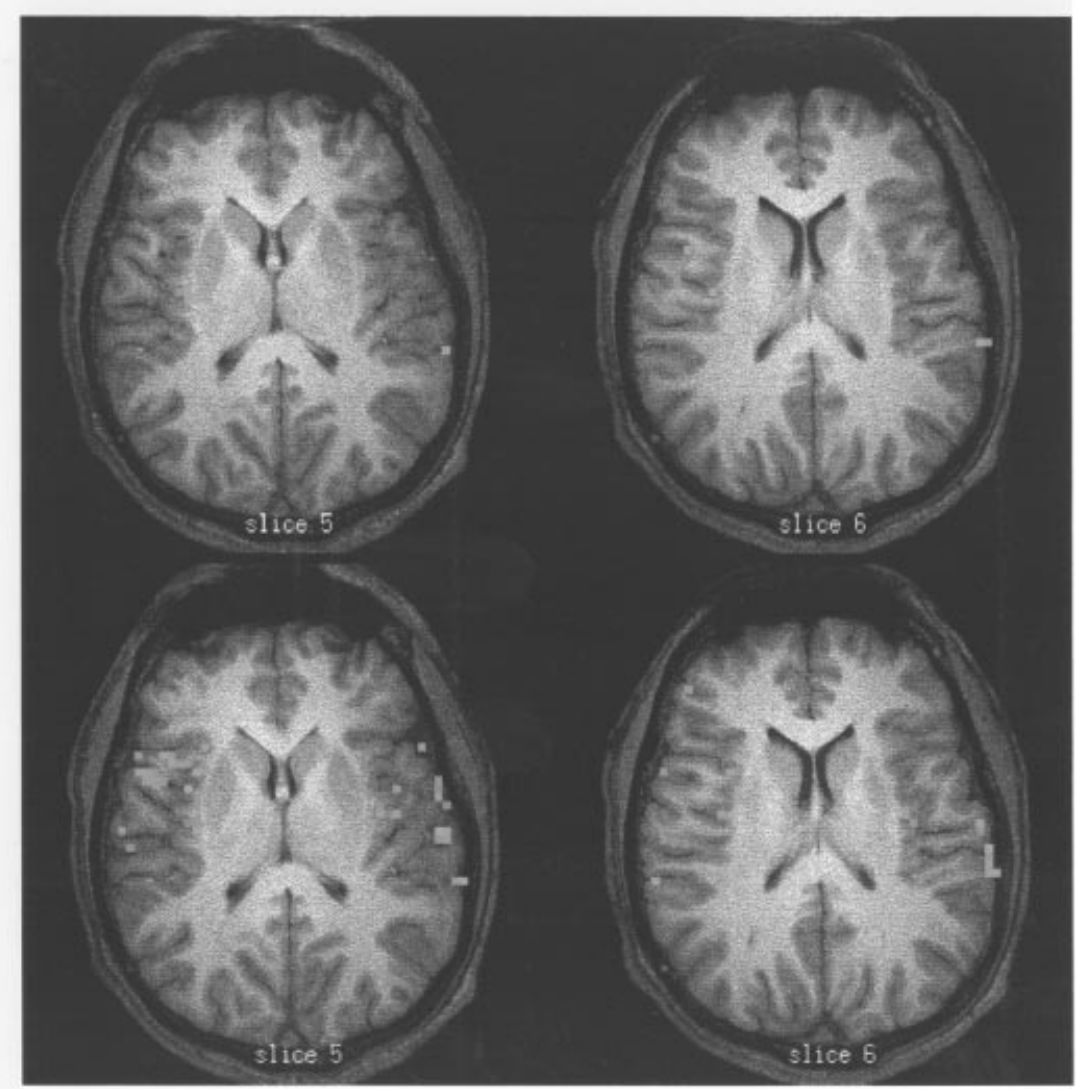

Figure 7. Activation maps calculated using SPW and PP (taste stimulation with Aspartame, same subject as in Fig. 6.) Using the PP, activation was observed in the left and right insula, the left and right inferior parts of the pre and post central gyri, and the right operculum. When the SPW was used to process the same data set, the number of activated clusters of pixels significantly dropped and activity could be located only in right insula. 
reasons, as pointed out by our perception study. When comparing pixel signals expected to exhibit comparable response shapes, this problem can be partly solved by crosscorrelating pixel signals with other recorded responses rather than with a theoretical reference waveform. However, the use of a 'perception profile' to detect brain activation might be a more efficient method. In our study, pixels extracted with PPs were more correlated to the MRI signal responses than those extracted with SPWs (Fig. 6), and were located in regions highly consistent with literature data concerning cortical taste projections. ${ }^{26,27}$ This observation suggests that the perception profile better represents the temporal evolution of cortical events occurring during the functional experiment than a stimulation-derived profile such as SPWs. The very high correlation of the MRI signal time course with the PP underlines that the MRI signal response has the same characteristics as the perception profile: a slow rise of signal, related to the slow rise of perceived taste intensity, a slow decrease of response after the end of effective taste stimulation related to slow rinsing of the stimulus, and eventually a remaining response level associated with perceptions not returning to baseline. The finger-span approach has been previously used in psychophysical measurements. ${ }^{20,21}$ In many ways this method seems to be well adapted to fMRI studies, because it is possible to introduce an amagnetic linear potentiometer in the magnet without disturbance of the fMRI acquisition. After a short training period our subjects were able to reliably indicate their level of perception. It should be pointed out that the absolute level of intensity, as indicated with the potentiometer, cannot be compared between individuals and should be used only as a relative index of perception. This limitation is not a concern when the fingerspan method is used with cross-correlation analysis, as autonormalization of both the MRI signal time-course and the reference waveform is performed. ${ }^{10}$ The motor task associated to the finger-span did not seem to interfere with the taste sensory task, which is not surprising, as subjects had to use the potentiometer both during the ON and OFF periods. Furthermore, control experiments with and without on-line finger-span recording did not show specific activation in motor areas associated with finger-spanning.

\section{CONCLUSION}

Several effects must be considered when dealing with the time shift of the MRI signal of activated regions. The effect of a slice-dependent time shift in multi-slice fMRI may be significant and must be taken into account before interpreting any time difference observed between activated regions located on different slices. Only then could the distribution of the residual time shifts be assessed, taking advantage of the whole brain imaging ability of multislice EPI series. Whether these variations can be ascribed to local hemodynamic effects, to a true chronometry of brain activation or to other effects remains to be investigated. Comparison with MEG/EEG or ERP studies would be most helpful in this respect. Some fMRI studies with a subsecond resolution have suggested that event-related fMRI responses could also be detected within a couple of seconds after the onset of a short stimulus. ${ }^{31}$ While the mechanism of this response is still under debate ${ }^{32}$ it may ultimately lead to a gain of one order of magnitude in the temporal resolution of fMRI.

One way to better understand the coupling between the observed MRI signal and the actual 'neuronal' response is to control perception using a finger-spanning technique during the fMRI acquisition. The use of this perception profile rather than an arbitrary waveform derived from the paradigm proves to be a powerful alternative to fMRI data processing, especially with chemical senses studies, when return to baseline is not always correlated to stimulus suppression. This approach should also be relevant to other kinds of stimulation task, as a realistic way of monitoring any expected or unexpected variations of the actual sensory input, which may interfere with higher order cognitive functions, such as attention, adaptation, fatigue or even variability of stimulus presentation.

\section{Acknowledgements}

This work was supported in part by CNRS ('GIS Cognition'), France.

\section{REFERENCES}

1. Kwong, K. K. Functional magnetic resonance imaging with echo planar imaging. Magn. Reson. Q. 11, 1-20 (1995).

2. Le Bihan, D. and Karni, A. Applications of magnetic resonance imaging to the study of human brain function. Current Opinion Neurobiol. 5, 231-237 (1995).

3. Binder, J. R., Jesmanowicz, A., Rao, S. M., Bandettini, P. A., Hammeke, T. A. and Hyde, J. S. Analysis of phase differences in periodic functional MRI activation data. 12th Annual Meeting of the Society of Magnetic Resonance in Medicine. Abstr., p. 1383 (1993).

4. Erb, M., Wildgruber, D., Klose, U., Britsch, P. and Grodd, W. $\mathrm{fMRI}$ of sequential activation of supplementary motor area and primary motor cortex during voluntary movement. 2nd Annual Meeting of Human Brain Mapping, p. S364 (1996).

5. Hykin, J., Coxon, R., Botwell, R., Glover, P. and Mansfield, P. Temporal differnces in functional activation between separate regions of the brain investigated with single shift imaging at 3.0 T. 15th Annual Meeting of the Society of Magnetic Resonance in Medicine. Abstr., p. 451 (1995).

6. Mansfield, P., Coxon, R. and Hykin, J. Echo-volumar Imaging (EVI) of the brain at $3.0 \mathrm{~T}$ : first normal volunteer and functional imaging results. J. Comput. Assist. Tomogr. 6, 847-852 (1995).
7. Bandettini, P. A., Wong, E. C., Binder, J. R., Rao, S. M. Jesmanowicz, A., Aaron, E. A., Lowry, T. F., Forster, H. V., Hinks, R. S., Hyde, J. S. Functional MR imaging using the BOLD approach: dynamic chararcteristics and data analysis methods. In Diffusion and Perfusion Magnetic Resonance Imaging: Applications to Functional MRI, ed. by D. Le Bihan. Raven Press, New York (1995).

8. Friston, K. J., Jezzard, P. and Turner, R. Analysis of Functional MRI Time-Series. Human Brain Mapping 1, 153-171 (1994).

9. Lee, A. T., Glover, G. H. and Meyer, C. H. Discrimination of large venous vessels in time-course spiral blood-oxygenlevel-dependant magnetic resonance functional neuro-imaging. Magn. Reson. Med. 33, 745-754 (1995).

10. Bandettini, P. A., Jesmanowicz, A., Wong, E. C. and Hyde, J. S. Processing strategies for time-course data sets in functional MRI of the human brain. Magn. Reson. Med. 30, 161-173 (1993).

11. Hellekant, G., Van der Wel, H., Brouwer, J. N., Glaser, D., Hard, C. and Roberts, T. W. Chorda tympani proper nerve responses to intra-arterial and surface stimulation of the tongue in rhesus monkey and rat. Chem. Senses 11(4), 557-566 (1986). 
12. Birch, G. G., Latymer, Z. and Hollaway, M. Intensity/time relationships in sweetness: evidence for a queue hypothesis in taste chemoreception. Chem. Senses 5(1), 63-78 (1980).

13. Pfaffmann, C., Bartoshuk, L., McBurney, D. H. Taste Psychophysics. In Handbook of Sensory Physiology, Vol. IV (2), pp. 75-101. Springer, Berlin (1971).

14. Kroeze, J. H. A. The taste of sodium chloride: masking and adaptation. Chem. Senses Flavour 3(4), 443-449 (1978).

15. Faurion, A. The physiology of sweet taste and molecular receptors. In Sweet-Taste Chemoreception, ed. by M. Math louti, J. A. Kanters and G. G. Birch, pp. 291-315. Elsevier, London (1993).

16. Faurion, A., Saito, S. and MacLeod, P. Sweet taste involves several distinct receptor mechanisms. Chem. Senses 5(2), 107-121 (1980).

17. Faurion, A. and Courchay, C. Taste as a highly discriminative system: a hamster intrapapillar single unit study with 18 compounds. Brain Res. 512, 317-332 (1990).

18. Le Bihan, D., Turner, R., Zeffiro, A., Cuenod, C. A., Jezzard, P. and Bonnerot, $V$. Activation of human primary visual cortex during visual recall: a magnetic resonance imaging study. Proc. Natl. Acad. Sci. (USA) 90, 11802-11805 (1993).

19. Leonardo, M., Fieldman, J. B., Sadato, R., Campbell, G., Ibanez, V., Cohen, L. G., Beiber, M. P., Pons, T., Turner, R., Le Bihan, D. and Hallett, M. A. Magnetic resonance functional neuroimaging study of cortical regions associated with motor task execution and motor ideation in humans. Human Brain Mapping 3, 83-92 (1995).

20. Guinard, J. X., Pangborn, R. M. and Shoemaker, C. F. Computerized procedure for time-intensity sensory measurements. J. Food Sci. 50(2), 543-544 and 546 (1985).

21. Yoshida, M. A microcomputer system to record and analyse time-intensity curves of sweetness. Chem. Senses 11(1), 105-118 (1986).

22. Cerf. B., Van de Moortele, P. F., Giacomini, E., MacLeod, P. Faurion, A. and Le Bihan, D. Correlation of perception to temporal variations of fMRI signal: a taste study. 4th Annual Meeting of the International Society of Magnetic Resonance in Medicine. Abstr., p. 280 (1996).

23. Paradis, A. L., Mangin, J. F., Bloch, I., Cornilleau-Peres, V., Moulines, E., Frouin, V. and Le Bihan, D. Detection of periodic signals in brain echo-planar functional images. 18th Annual International Conference of the IEEE Engineering in Medicine and Biology Society (IEEE-EMBS). Abstr., p. 3.2.2-2 (1996)

24. Kwong, K. K., Belliveau, J. W. and Chesler, D. A. Dynamic magnetic resonance imaging of human brain activity during primary sensory stimulation. Proc. Natl Acad. Sci. (USA) 89, 5675-5679 (1992).

25. Rao, S. M., Binder, J. R., Brandettini, P. A., Hammeke, T. A., Yetkin, F. Z., Jesmanowicz, A., Lisk, L. M., Morris, G. L. Mueller, W. M., Estkowski, L. D., Wong, E. C., Haughton, V. M. and Hyde, J. S. Functional magnetic resonance imaging of complex human movements. Neurology 43, 2311-2318 (1993)

26. Ogawa, H. Gustatory cortex of primates: anatomy and physiology. Neurosci. Res. 20, 1-13 (1994).

27. Norgren, R. Gustatory system. In The Human Nervous System, ed. by G. Paxinos, pp. 845-861. Academic Press, New York (1990).

28. Sereno, M. I., Dale, A. M., Reppas, J. B., Kwong, K. K., Brady, T. J., Rosen, B. R. and Tootell, R. B. H. Borders of multiple visual areas in humans revealed by functional magnetic resonance imaging. Science 268, 889-893 (1995).

29. Savoy, R. L., Bandettini, P. A., O'Craven, K. M., Kwong, K. K. Davis, T. L., Baker, J. R., Weisskoff, R. M. and Rosen, B. R. Pushing the temporal resolution of fMRI: studies of very brief visual stimuli, onset variability and asynchrony, and stimulus-correlated changes in noise. 15th Annual Meeting of the Society of Magnetic Resonance in Medicine. Abstr., p. 450 (1995).

30. Lang, W., Lang, M., Podreka, I., Uhl, F., Suess, E., Müller, C. and Deecke, L. DC-potential shifts and regional cerebral blood flow reveal frontal cortex involvement in human visuomotor learning. Expl. Brain Res. 71, 353-364 (1988).

31. Ernst, T. and Hennig, J. Observation of a fast response in functional MR. Magn. Reson. Med. 32, 146-149 (1994).

32. Merboldt, K. D., Krüger, G., Hänicke, W., Kleinschnmidt, A. and Frahm, J. Functional MRI of human brain activation combining high spatial and temporal resolution by a CINE FLASH technique. Magn. Reson. Med. 34, 639-644 (1995). 